\title{
SENTENCIAS ADITIVAS Y LOS PRINCIPALES CONFLICTOS EN EL ÁMBITO DE LA INTERPRETACIÓN CONSTITUCIONAL
}

\author{
Jorge Alberto Taylor Santos ${ }^{1}$ \\ Angelica Saraí Santos Hernández ${ }^{2}$
}

DOI: https://doi.org/10.5377//rd.v42i1.12936

\section{RESUMEN:}

La interpretación conforme y los juicios de ponderación son sin duda dos recursos interpretativos de suma importancia al momento de llevar a cabo ejercicios de interpretación constitucional, los cuales, y partiendo de la premisa que la constitución en su condición de norma jurídica suprema de los ordenamientos jurídicos puede aplicarse de forma directa a la mayoría de las actuaciones y vida jurídica de los países. Sin embargo, el reto que supone el desarrollo de estos principios de interpretación, no son pocos y a menudo conllevan graves riesgos en cuanto a la extralimitación que pueda derivarse en su aplicación. Aun y cuando existen riesgos, la Constitución hondureña contiene cláusulas de apertura que sumadas a un Sistema de integración de Derechos Humanos tan fuerte como supone el Sistema Interamericano de Derechos Humanos, y el Sistema Universal de Naciones Unidas a través de sus procedimientos especiales, pone a los juristas en una situación crucial, puesto que devienen obligados en la administración de justicia, lo que conlleva forzosamente un ejercicio interpretativo de la Constitución, a menudo en temas de alta controversia por su fuerte contenido político y de valores.

PALABRAS CLAVE: Sentencias Aditivas, Ponderación, Interpretación Constitucional, Mutación Constitucional.

Fecha de recepción: $31 / 8 / 21$

Fecha de aprobación: 1/11/2021

1 Máster en Derecho Constitucional, Jefe Unidad de Asesoría Legal, Dirección de Niñez, Adolescencia y Familia (DINAF) Correo Electrónico: jorge taylorsantos@hotmail.com

2 Máster en Derecho Constitucional, Secretaría Ejecutiva Programa Presidencial Honduras 2020,

Correo electrónico: ansasah@yahoo.com 


\title{
ADDITIVE JUDGMENTS AND MAJOR CONFLICTS IN THE FIELD OF CONSTITUTIONAL INTERPRETATION
}

\author{
Jorge Alberto Taylor Santos ${ }^{3}$ \\ Angelica Saraí Santos Hernández ${ }^{4}$
}

DOI:https://doi.org/10.5377//rd.v42i1.12936

\begin{abstract}
:
The consistent interpretation and weighting trials are undoubtedly two interpretative remedies of paramount importance when carrying out exercises of constitutional interpretation, which, and on the basis of the premise that the constitution as the supreme legal norm of legal systems can be applied directly to most actions and legal life of countries. However, the challenge of developing these principles of interpretation is few and often carries serious risks as to the overreach that may arise in their application. While there are risks, the Honduran Constitution contains opening clauses that add to a System of Human Rights integration as strong as the Inter-American System of Human Rights, and the United Nations Universal System through its special procedures, puts jurists in a crucial situation, since they become forced into the administration of justice, which necessarily entails an interpretive exercise of the Constitution, often on issues of high controversy for its strong political content and values.
\end{abstract}

KEY WORDS: Additive Sentences, Weighting, Constitutional Interpretation, Constitutional Mutation

Date received: $8 / 31 / 21$

Approval Date: 11/1/2021

3 Master in Constitutional Law, Head of the Legal Advisory Unit, Directorate for Children, Adolescents and Family (DINAF) Email: jorge taylorsantos@hotmail.com

4 Master in Constitutional Law, Executive Secretariat of the Presidential Program Honduras 2020,

Email: ansasah@yahoo.com 


\section{INTRODUCCIÓN}

Luigi Ferrajoli en su obra Principia Iuris, señala tres tipos de filosofías que revolucionaron el derecho, cada una reemplazante de la anterior, la primera el ius-naturalismo, característico de los ordenamientos pre-modernos, los cuales consideran que las normas existen por su contenido al considerárseles intrínsecamente justo, razonable o acreditado por precedentes, practicas consuetudinarias o similares; el iuspositivismo el cual confiere un carácter positivo y normodinámico a través del principio de legalidad formal o de mera legalidad, conforme al cual una norma existe y es válida únicamente por la forma legal de su producción, a su vez establecida por una norma deóntica positiva superior a ella; y por último el ius-constitucionalismo que cuenta con una característica estructural más, el principio de legalidad sustancial o de estricta legalidad, conforme al cual una norma es válida, además de vigente, solo si sus contenidos contrastan con los principios y los derechos fundamentales establecidos por la constitución (Ferrajoli y Andrés Ibáñez, DL 2011).

En la actualidad, estamos ante el cambio de paradigma por el modelo ius- constitucionalista, en el cual aparece el término Constitución que va más allá de una norma jurídica, abarcando hasta la propia organización y límites del Estado, dando respuesta a ciertos principios. La constitución como nueva protagonista, y la propia indeterminación de muchos preceptos de índole constitucional, implica un verdadero desafío para los juristas, quienes deben acudir a la interpretación como mecanismo obligatorio para la adecuada resolución de los desafíos y problemas constitucionales.

\section{METODOLOGÍA}

La metodología empleada para el desarrollo del presente artículo consta de tres métodos: inductivo proceso mediante el cual, a partir de estudio de casos particulares, se obtienen conclusiones o leyes universales que explican o relacionan los fenómenos estudiados, es decir, se intenta ordenar la observación tratando de extraer conclusiones de carácter universal desde la acumulación de datos particulares; Deductivo, proceso mediante el cual se intentan obtener conclusiones de carácter particular, a través de una conclusión general, por cuanto al partir de principios generales $y$, con la ayuda de una serie de reglas de inferencia, se logra desarrollar principios secundarios que ayudan a obtener las respuestas buscadas ; y dialéctico método que investiga la verdad mediante el examen crítico de las percepciones y teorías, mediante el intercambio de proposiciones (tesis) y contraproposiciones (antítesis), resolviendo la contradicción a través de la formulación de una síntesis final (conclusión) (Münch y Angeles, 1990).

\section{EL ESTADO CONSTITUCIONAL DE DERECHO Y LA INTERPRETACIÓN CONSTITUCIONAL}

El Estado Constitucional de Derecho, se rige bajo el principio de la supremacía de la Constitución sobre la ley, subordinándolas al texto constitucional al ser el texto constitucional la norma suprema del ordenamiento jurídico, misma que fue otorgada por el pueblo de forma directa a través de la asamblea constituyente. Esta característica diferencia a la Constitución de un país de las leyes las cuales son un producto del trabajo del legislador en atención al poder delegado en este Poder del Estado, por el pueblo. 
Sin embargo, y para los fines de nuestro análisis, observaremos más adelante como en base al principio pro homine o pro-persona, y el control de convencionalidad, a menudo dicha jerarquía que tan conveniente nos resultaba para dilucidar antinomias, resulta subordinada a la aplicación de la norma más favorable y garante.

Otra característica interesante del Estado Constitucional de Derecho es en atención a su división de poderes, ya que el rol de estos varia respecto a el Estado de Derecho en donde el legislativo tenía un poder casi ilimitado, y un poder judicial muy debilitado, amparado en el principio de supremacía de la ley. El Estado Constitucional agrega garantías para el cumplimiento de las disposiciones constitucionales, bajo la premisa de que los poderes constituidos son creados por el constituyente, quien estableció sus competencias y límites en el texto constitucional, y el respeto a los mismos garantizados a través de la jurisdicción constitucional, es lo que viene a garantizar esa división de poderes, lo que a su vez, trae como consecuencia directa la limitante a los poderes en adoptar decisiones absolutas, mismas que tendrán validez siempre y cuando estén armonizadas con el texto constitucional y respetando los límites establecidos en esta (Fernández Segado y Silva, 2003).

Un elemento importante a resaltar es que todos los poderes públicos y de los particulares se encuentras sometidos a la Constitución, no pudiendo extralimitarse de aquellos parámetros fijados en el texto constitucional, lo que a su vez resulta vinculante para la ciudadanía quienes debe cumplir con los deberes establecidos en la Constitución. Otro elemento de gran importancia, relacionado al primer elemento del Estado Constitucional, como ya dijimos anteriormente, reconoce la naturaleza jurídica de la constitución como norma sustantiva cualitativamente superior a la cual se puede recurrir por la vía jurisdiccional, y como resultado directo el establecimiento de dispositivos de control cuya finalidad es garantizar los límites impuestos por la constitución, siendo el más importante de dichos controles la existencia de una jurisdicción especializada en materia constitucional, mediante la cual se determine la constitucionalidad de los actos de los entes estatales y los particulares .

No obstante, es de recalcar que recientemente existen teorías constitucionales contrapuestas en relación a quien debería ser quien defienda soberanamente la Constitución a través de su interpretación, teniendo por una parte posturas que defienden el rol importante del juez y el judicial review en el Estado Constitucional, como las teorías de John Elster quien concibe al poder judicial como el mástil de Ulises, es decir, el encargado de cumplir las limitantes auto-impuestas por el pueblo y consagradas en los textos constitucionales, hasta el debate del Constitucionalismo Popular y su oposición a la supremacía judicial, criticando el hecho de que invaliden las acciones de los poderes con legitimidad plebiscitaria (Ejecutivo y Judicial), por aquel que ostenta menos representación, lo que implicaría la necesidad de devolver dicho poder de interpretación y defensa a donde se originó "el pueblo", a través de procesos de discusión popular y así proveer de una fuente de legitimidad para el derecho constitucional.

El punto intermedio entre estas teorías contrapuestas, a nuestro criterio, y por consiguiente la más acertada, es justamente la planteada por Peter Häberle con su teoría cultural de la Constitución, quien propone que el estudio de la Constitución debe ser como una ciencia cultural, debiendo considerar las 
particularidades históricas elaboradas por los pueblos a los cuales las constituciones regulan, y la categoría Tiempo (diacrónico- experiencia histórica, sincrónico- proceso constituyente), y espacial (aquellos elementos que los actos constituyente han aportado), para entender al Estado Constitucional, convirtiendo de esta forma las convicciones de una sociedad, en conducta y normas de garantía a la vez y por lo tanto no debería haber un numero cerrado de intérpretes de la constitución. Esta última teoría, nos parece muy acertada ya que como se puede observar con los ejemplos del nuevo constitucionalismo latinoamericano, la Teoría del Estado Constitucional en cuanto al termino constitución, se va a ver completada al ser el canal o medio mediante el cual el poder constituyente expresara su voluntad (Häberle, 2002).

Todos los sistemas jurídicos constitucionales han conocido polémicas por algunas interpretaciones muy cuestionables de la Constitución. Honduras, no ha sido la excepción con su sentencia de 22 de abril de 2015 (RI 02432015). Sin perjuicio de lo anterior, como veremos a en el presente artículo las discrepancias y el impacto que generan muchas veces las decisiones adoptadas por estos órganos, en parte resulta justificable dado el delicado rol del Tribunal Constitucional, y en el caso hondureño de la Sala Constitucional, van generando una transformación del sistema con ocasión de la interpretación de la Constitución. Dicha transformación a través de la interpretación constitucional, tiene su causa por una parte en la fuerza normativa de la Constitución, la obliga a todos los operadores jurídicos a hacerla vigente en todos los procesos, y por otro lado en la alta indeterminación de muchos de los preceptos constitucionales, lo que genera una amplia discrecionalidad interpretativa a mano de los jueces.

\section{LA INTERPRETACIÓN CONSTITUCIONAL}

Comenzamos afirmando que las transformaciones a raíz de la interpretación constitucional se llevan a cabo a través de dos recursos interpretativos los que, si bien a menudo no tienen un desarrollo en el texto constitucional, se llevan a cabo en el ejercicio del rol del tribunal constitucional y que doctrinariamente se conocen como: el criterio de la Interpretación Conforme y por otro el Juicio de Ponderación. Estos métodos pueden dar como resultado la transformación de la magistratura constitucional a sujeto político, generando así desbalances en el delicado equilibrio entre el juicio político y el juicio de constitucionalidad (Ferrer, 2011, p. 343).

Antes de continuar, es importante precisar los límites del juicio de constitucionalidad, mismos que tienen que ver con la manera de entender la Constitución y paralelamente con la forma en que concebimos las relaciones entre Constitución y ley. En primer lugar, podemos afirmar que la Constitución es la norma Suprema del Sistema que se impone a todos los Poderes. A partir de aquí, surgen dos lecturas de la supremacía constitucional, en primer lugar, una lectura "constitucionalista" en la cual se concebiría a la Constitución como una norma que encierra un proyecto político y social bien articulado y cerrado. Bajo esta primera premisa, la Constitución debería contener soluciones para la mayor parte de los problemas sociales, aunque no siempre claras por lo que se tendría que recurrir a a complicadas técnicas argumentativas, o interpretativas; sin embargo, la Constitución si contemplaría soluciones para todos los conflictos importantes, siendo en este modelo los jueces los que desempeñan un papel fundamental en la concreción de las normas en cada momento concreto a fin de configurar el sistema (Martínez, 
2016). Sobre todo, los jueces constitucionales a la hora de controlar la constitucionalidad de la ley, pero también los jueces ordinarios que pueden aplicar la Constitución en detrimento de la ley si fuera necesario (Julios-Campuzano y Pérez Luño).

Respecto a la Segunda Lectura denominada "democrática", la Constitución no encierra un proyecto social o político perfectamente acabado. Es decir, no predetermina la solución para todos los conflictos, sino que se limita simplemente a fijar las reglas del juego político. Estas reglas serian la de competencia política y el marco abierto de valores en el que el legislador puede moverse. Así, dentro de ese marco de valores, cabrían opciones políticas muy variadas, centrándose en la figura de un legislador democrático, quien tiene un papel fundamental al determinar qué normas, entre las constitucionalmente posibles, conforman el sistema político histórico concreto. En este modelo, el juez ordinario viene sujeto al principio de legalidad y el juez constitucional sólo debe declarar inconstitucional la ley cuando ésta rebase el marco de posibilidades políticas que la Constitución permite naturalmente (JuliosCampuzano y Pérez Luño).

Ambas lecturas (constitucionalista / democrática), marcan la pauta en la que los sistemas constitucionales contemporáneos se ubican y resultan validas en gran medida. No obstante, el principio democrático exigiría aproximarse más a la segunda visión de la Constitución, ya que la primera deriva en un sistema más constitucionalista, pero menos democrático. Así, la lectura democrática de la Constitución, exigible desde un punto de vista del respeto del principio democrático, se refleja en el lugar que la ley deberá ocupar en el sistema, ya que la misma por cuanto expresión del principio democrático, sigue teniendo en el sistema constitucional una autónoma razón de ser y un ámbito propio que el juicio de constitucionalidad no puede invadir (Julios-Campuzano y Pérez Luño).

De lo desarrollado en el párrafo anterior derivan dos consecuencias para los jueces, la primera es que los jueces ordinarios vienen sujetos al principio de legalidad sin que con la excusa de hacer valer la Constitución, en el caso del que están conociendo, puedan invadir el espacio de la política y el juez constitucional debe realizar su función de control de constitucionalidad de la ley, con una separación rigurosa entre las cuestiones políticas y las cuestiones de constitucionalidad (Vigo, 2017). En definitiva, la función del Tribunal Constitucional jamás debe entenderse como una sustitución del Poder Legislativo, el cual goza de una indudable libertad política, tratando de fijar una mejor ley desde la perspectiva constitucional, sino tan sólo la de eliminar aquellas leyes que resulten intolerables. Por consiguiente, la tarea del Tribunal Constitucional, es la de controlar la constitucionalidad de la ley, y no debe entrar a valorar los móviles políticos que han animado al legislador y mucho menos debe sugerir o directamente imponer al legislador una opción política determinada (Espín Templado y Díaz Revorio, 2000).

\subsection{El Principio de Interpretación Conforme y el Juicio de Ponderación}

Las dificultades para mantener una separación rigurosa entre juicio político y juicio de constitucionalidad, puede examinarse a partir de las particularidades de la interpretación por medio del principio de interpretación conforme y el juicio de ponderación (Queralt Jiménez, 2008). Ambas técnicas conllevan un riesgo claro 
de intromisión de los jueces, en el ámbito de la política y la afectación del principio democrático; asimismo, conlleva el riesgo de deslegitimación de la función judicial, ante el riesgo de las extralimitaciones del juicio de constitucionalidad que se lleva a cabo con ocasión del principio de interpretación conforme.

Previo a continuar, es importante precisar que la interpretación conforme es un principio que establece que, entre las interpretaciones plausibles de una ley, sólo son legítimas aquellas que se acomoden a la Constitución, es decir, que no contradigan la misma. Derivándose dicho principio interpretativo, de la primacía constitucional, yaque dentro delasinterpretaciones plausibles de la ley, se discrimina entre las que son compatibles con la Constitución y aquellas que no lo son (Kuhlen, 2012). En ese orden de idas, la interpretación conforme procedería a llevar a cabo un ejercicio de discriminación entre las interpretaciones plausibles de la ley, es decir las que sean compatibles con su semántica en conjunción obviamente con la sintaxis y con la pragmática.

En virtud de lo expuesto en el párrafo anterior $\mathrm{y}$, bajo el pretexto de la interpretación conforme, los jueces a menudo retuercen arbitrariamente el sentido de la ley, pasando de esta forma a ejercer funciones políticas al dictar sentencias con pronunciamientos interpretativos (Díaz Revorio, 2001). Estas sentencias interpretativas, son aquellas en las que el Tribunal Constitucional declara la constitucionalidad del precepto legal impugnado, en la medida dicen que se interprete en el sentido que el Tribunal considera conforme a la Constitución. En definitiva, cuando un juez constitucional dicta un pronunciamiento interpretativo, lo que señala es que existen otras posibilidades de interpretar la ley, diferentes a la que se está rechazando, y que de acuerdo con esas otras interpretaciones plausibles que la propia sentencia proporciona, la ley resultaría compatible con la Constitución, como si fuese una manera de corrección.

Las sentencias interpretativas a menudo, debido a su contenido altamente técnico, no tienen nada de reprobable al conjugar la primacía de la Constitución, con la conservación de las leyes (Díaz Revorio, 2001). De esta forma resulta distinta con el legislador, en la medida en que se está imponiendo la Constitución, al mismo tiempo que se está respetando la libertad de configuración normativa que el legislador tiene. No obstante, las sentencias interpretativas entrañan un riesgo evidente, ya que con la excusa de la interpretación conforme el juez constitucional podría terminar haciendo una interpretación de la ley extraña que no se deduce de su texto y en este caso se estarían traspasando los límites de la interpretación conforme, misma que está marcada por las interpretaciones plausibles de la ley (Balaguer Callejón, 1997).

Al traspasar estos límites, se estaría ante una simple alteración del ordenamiento invadiendo el ámbito que la Constitución reserva al legislador, siendo este tipo de actuaciones cuestionables en la doctrina jurídica, denominándose este tipo de sentencias como "sentencias o pronunciamientos manipulativos" (Bonifacio y López Pina, 1987). Este tipo de actuaciones que cambian un ordenamiento jurídico por la vía del control de constitucionalidad, tienen lugar cuando ninguna de las interpretaciones plausibles del precepto legal impugnado, permite mantener la constitucionalidad de la ley; no obstante, el Tribunal Constitucional no considera conveniente o adecuado anular el precepto legal (Canosa Usera, 1988). 
En estos casos, el Tribunal tiene una posibilidad de salvar la constitucionalidad de la ley al manipular la interpretación de la misma. Forzando las posibilidades interpretativas de la ley (interpretación contra legem), a fin de que esta ley resulte compatible con la Constitución. Las manipulaciones que pueden llevarse a cabo con la excusa de la interpretación conforme, son de muy distinto tipo, resultando más evidentes las que tienen lugar a través de lo que también se ha denominado en la doctrina "sentencias aditivas" (Díaz Revorio, 2001).

\subsection{Las Sentencias Aditivas y sus Riesgos}

Las Sentencias Aditivas, consisten en hacer simplemente una interpretación extensiva del ámbito de aplicación del precepto legal que sea impugnado, a fin de conformarlo a la Constitución. De esta manera, tras la interpretación que el Tribunal Constitucional realiza a la ley, resulta aplicable a más supuestos de los comprendidos en abstracto por el enunciado legal.

Este tipo de pronunciamientos aditivos son bastante comunes en los Tribunales Constitucionales, un ejemplo de ello son las sentencias aditivas emitidas por el Tribunal Constitucional español, los que han permitido durante el tiempo en que no había cobertura legal para estas cuestiones extender, a las parejas de mismo género, muchos de los derechos que la ley solamente concedía a los matrimonios naturales o entre personas de distinto género (Martínez Zorrilla, 2007). Estas sentencias manipulativas en general y aditivas en particular, evitan la arbitrariedad al tener una buena y sólida justificación constitucional, motivo por el cual la necesidad de su censura no resulta evidente para todos al considerarse debidamente justificada (Mendonca, 2003).
Un ejemplo claro de sentencia aditiva se produce en el ámbito de lo que se denomina la inconstitucionalidad por omisión, es decir cuando se impugna una ley no por lo que dice, sino justamente por lo que no dice (Villaverde Menéndez, 1997). En este sentido, la inconstitucionalidad opera en la medida en que la Ley, no está previendo algo necesario, por lo que se impugna, por ejemplo, cuando el legislador ha concedido una serie de derechos, de ventajas o beneficios a una clase de sujetos, excluyendo a otros sujetos. En estos casos, el Tribunal entiende que la ley es inconstitucional al privar a un grupo de sujetos de derechos, vulnerado el principio de igualdad. Normalmente, en estos casos, el tribunal tendría que anular la ley declarando su inconstitucionalidad. Sin embargo, esta estima que, al anularse, causaría perjuicios inmediatos a todos aquellos a los que la ley confería derechos. En ese sentido, ante los prejuicios que derivarían de la simple ley, el Tribunal opta por reparar la inconstitucionalidad, añadiendo por vía interpretativa la regulación que falta.

El resultado es dejar intacta la Ley, imponiendo una interpretación de la misma que extienda los derechos al grupo discriminado. Estas sentencias, aunque se dicten para salvar la igualdad presuntamente vulnerada por esa omisión legislativa, crean, aunque sea por vía interpretativa, una nueva norma que en adelante es de obligado cumplimiento para los operadores jurídicos, pero que, sin embargo, no ha sido la intención o espíritu del legislador.

Este tipo de "nuevas normas", al no haber sido establecidas por el legislador, implican que el Tribunal se esté comportando como un auténtico legislador positivo, y por consiguiente haciendo valoraciones políticas (Márquez Luzardo, 2014). Esta actuación de los tribunales constitucionales, 
puede llegar a ser reprobable en primer lugar, ya que la sentencia en si puede originar una situación de inseguridad jurídica, al reconocer al grupo de sujetos discriminados derechos que no existían. Pero, además, faltaría la regulación de su ejercicio y, sobre todo, en muchas ocasiones, las previsiones económicas que son necesarias para para su satisfacción.

Lo anterior, supone un verdadero problema puesto que debemos considerar que las regulaciones y previsiones económicas y sociales son necesarias a fin de evitar que el ejercicio de los derechos, que ahora se reconocen en la sentencia, se desborden más allá de lo previsto y deseable por esa interpretación constitucional, pero al mismo tiempo el Tribunal no puede hacer esta regulación al ser este un órgano torpe para legislar positivamente al no ser esta su función constitucional, al mismo tiempo que arrebata al legislador competencias que le son propias. En ese sentido, tendríamos que evaluar si el legislador ante la alternativa de emitir una legislación completamente distinta, para todos quizás podría preferir esta opción al resultar más pertinente para los fines perseguidos.

La interpretación realizada por el Tribunal a fin de eliminar la inconstitucionalidad la interpretación o la manipulación, es una forma de arrebatar al legislador su libertad de configuración normativa. Este tipo de pronunciamientos, pone de relieve que la interpretación conforme es una técnica de muy delicado manejo que obliga al Tribunal Constitucional a realizar constantemente un esfuerzo auto inhibitorio a fin de no terminar transformándose en un legislador positivo, por lo demás más allá de todos estos pronunciamientos, dicha intromisión de la justicia constitucional en cuestiones políticas viene muchas veces propiciada por la indeterminación del propio texto constitucional (Niembro Ortega et al., 2012). Texto en el que son frecuentes cláusulas muy abiertas o de fuerte contenido valorativo, situación que no ocurre cuando en una cuestión de inconstitucionalidad, las disposiciones están altamente formalizadas $\mathrm{y} \mathrm{su}$ interpretación no ofrece casi ningún problema. A manera de ejemplo no habría lugar a cuestionar la actuación de un Tribunal Constitucional si el Tribunal invalidase una ley que establece la pena de muerte para ciertos delitos, sencillamente porque la interpretación del precepto constitucional que prescribe la pena de muerte no plantea ninguna duda el Tribunal al establecerse la prohibición de pena de muerte en la Constitución Hondureña.

En estos casos, el Tribunal únicamente habría interpretado la Constitución, aplicando la Constitución en un supuesto claro; sin embargo cuando lo que está en juegos es un precepto constitucional de significado altamente indeterminado, por ejemplo cuando no se es claro qué es lo que la Constitución permite o prohíbe en un determinado punto, entonces el juicio de constitucionalidad resulta notablemente discrecional, y en algunos casos resulta tan discrecional que pareciera obligada la transición del Tribunal Constitucional a convertirse en un sujeto político (María Luisa Balaguer Callejón).

Los casos más delicados tienen lugar cuando la indeterminación afecta a principios o valores constitucionales cuya interpretación es socialmente muy controvertida, siendo el Tribunal Constitucional quien termina fijando directamente el significado de estos principios o valores de manera intolerable desde la perspectiva del principio democrático (Díaz Revorio, 2001). Por ejemplo, no cabe duda de que, si una ley regula la eutanasia y ha sido votada por una amplísima mayoría en el Parlamento, y esta fue 
invalidada por un Tribunal Constitucional por vulnerar el derecho a la vida, seria justificable cuestionar quién es el Tribunal para imponer su interpretación del derecho a la vida por encima de la que ha hecho el legislador democrático. De igual forma, si el tribunal en ese mismo supuesto declarase la constitucionalidad de la ley, sin más, estaría también alimentando la idea de que la Constitución exige o impone esa interpretación del derecho a la vida que se ha hecho en la ley y que en adelante ninguna otra mayoría política distinta podría modificar esa interpretación o esa legislación (Atienza, 2013).

Es en ese sentido, que todas estas actuaciones son particularmente polémicas y delicadas ya que alimentan la objeción antidemocrática a la que siempre se enfrenta la justicia constitucional, es decir la que se sustancia en la tesis de que las generaciones pasadas no pueden vincular a las generaciones futuras (Guastini, 2008). Muchos autores, consideran que el problema que plantean este tipo de pronunciamientos de cuestiones constitucionales socialmente muy controvertidas, tal vez podría salvarse si el Tribunal adoptará una especie de solución intermedia una solución consistente en declarar válida la ley, pero dejando al propio tiempo abierta la reversibilidad de su decisión. En ese sentido, por razones democráticas y habida cuenta la duda, es preferible diferir la cuestión al criterio de la mayoría, declarando válida la ley por el momento, pero reconociendo que los argumentos que se invocaron por la minoría que impugnó la ley, tienen también peso suficiente como para dudar de la constitucionalidad de la misma.

\subsection{Interpretaciónen los Sistemas Constitucionales}

Otra gran particularidad de la interpretación en los sistemas constitucionales, qué tiene que ver también con el fuerte contenido material o sustantivo de nuestras constituciones y la indeterminación de la Constitución, se manifiesta de modo particular cuando se producen colisiones entre preceptos constitucionales, especialmente en materia de derechos y libertades (España, 1992). Esto se resuelve a través del juicio de ponderación, sin embargo, este juicio podría dar también lugar a la intromisión de la justicia ordinaria en el ámbito de la política, soslayando el principio democrático y el de separación de poderes en el que se asienta todo el esquema constitucional moderno (Jestaedt y Montealegre Lynett, 2008).

Debemos así, partir de la premisa que las constituciones no son simples cartas formales de distribución del poder, sino que reconocen también un catálogo de derechos y principios de Justicia, que son el reflejo de las concepciones morales vigentes en la sociedad de que se trate. Dicho conjunto de principios de Justicia, podrían considerarse como una realidad moral positivizada, reconociendo el valor de la libertad, de la igualdad, de la vida, y el pluralismo político. De esta forma se reconoce el valor de la ideología de la libertad ideológica de pensamiento, a la intimidad, al honor y muchos otros valores de muy distinto tipo. Ahora bien precisamente porque la justificación de todos estos principios son en realidad purificación de valores morales, su formulación se hace mediante frases muy contundentes, pero que padecen de una altísima indeterminación (García Manrique, 2007).

Es así, que en torno a estas normas (a las que denominaremos principios), pueden surgir dudas no sobre la fortaleza de los valores protegidos, pero sí sobre alcance de estos valores. Por ejemplo, la premisa de "todos tienen derecho a la vida", en un primer momento puede parecer bastante clara, e 
incluso ser considerada así por un gran consenso de estudiosos en una primera aproximación al principio. Se podría entonces concluir que tanto el significado y alcance resultado claro, así en muchos supuestos queda claro que se protege la vida frente a la amenaza de los particulares y por supuesto frente al Estado, derivando así la prohibición de la tortura, pena de muerte y por consiguiente la justificación de una parte importante del Código Penal, el cual tipifica los delitos contra la vida (Fernández de Casadevante Romani, 2020). No obstante, esta premisa y consenso generalizado, resulta en especial problemática para principios como la idea del aborto, el cual supondría un problema particular ya que, bajo la premisa del derecho y protección a la vida, se plantea en un primer momento de manera absoluta, protegiendo a la persona desde el mismo momento de su concepción y por consiguiente el aborto sería incompatible con esta visión. Es así, como tendrían que surgir diferentes teorías de protección gradual dependiendo de la etapa del desarrollo embrionario y por tanto el aborto hasta cierto momento puede estar permitido (Parejo Alfonso, DL 1990). Es así, como otros casos importantes salen a la luz, temas sensibles como la clonación no reproductiva, tiene opiniones conflictivas entre detractores y quienes están a favor en las implicaciones de esta, como por ejemplo la destrucción de embriones clonados, sobre los cuales se plantean importantes debates respecto a si se protege la vida, al punto de impedir que los embriones sobrantes del uso de las técnicas de reproducción asistida, sean donados para fines investigativos. Otro caso, es respecto a si se debe proteger la vida incluso frente a su titular, en cuyo caso estaríamos proscribiendo constitucionalmente también la eutanasia, bien se protege frente a todos pero no frente a su titular (Instituto Iberoamericano de Derecho Constitucional, 2009).
La expresión más genuina de todas estas dudas se presenta por supuesto articulada a través de los conflictos entre derechos, por ejemplo, si está en debate la legitimación de una intervención de las comunicaciones que se ha llevado a cabo en el curso de una investigación judicial en la persecución de un delito grave, entraría en debate dos valores, y por lo tanto tendríamos que definir claramente qué valor es al que hay que dar preferencia. El valor de la privacidad en las comunicaciones que es un valor esencial, o la seguridad pública y por consiguiente al interés del Estado en la persecución del delito y el castigo del delincuente. Volviendo al caso de la eutanasia, vale la pena considerar si esta debe despenalizarse, o incluso regularla tal y como sugiere el reconocimiento del valor de la autonomía de los sujetos, o bien, por el contrario, debe seguir siendo criminalizada según se desprende del reconocimiento del derecho a la vida y su interpretación como derecho. Deber que exige por tanto una protección absoluta (Atienza, 2013)

Es evidente que los conflictos entre principios constitucionales, no admiten soluciones generales, por ejemplo no todos los conflictos entre la libertad y la seguridad se tienen que resolver siempre de la misma manera, ello así aunque sólo sea porque todos estos derechos y principios tienen por así decirlo una misma dignidad constitucional, por tanto no sería constitucionalmente lícito otorgar siempre y en todo caso preferencia a uno de ellos sobre los demás (Wolfe et al., 1991). En ese sentido, la solución para estos conflictos pasa necesariamente por construir una regla que otorgue la preferencia a uno de los derechos en conflicto. En los supuestos donde se articula esta preferencia de uno de los derechos enfrentados, es lo que se ha denominado en terminología como ponderación o juicio de proporcionalidad. 


\subsection{Ponderación o Juicio de Proporcionalidad}

El juicio de proporcionalidad implica decidir si la importancia de las razones para proteger un cierto derecho justifica o compensa la intensidad con la que se está afectando o minando el otro derecho en conflicto. En ese sentido la ponderación en realidad estimula la argumentación moral o en todo caso un tipo de argumentación muy abierta. La ponderación se convierte así en una potentísima herramienta argumentativa en un ámbito de tan intensa discrecionalidad, donde es necesario hacer una argumentación esmerada, con buenas razones y transparente (Jestaedt y Montealegre Lynett, 2008).

La ponderación, además, es un excelente mecanismo de control, a fin de que el poder discrecional no se convierta en un poder arbitrario; sin embargo, en muchos casos resulta inevitable que persista la subjetividad del juicio de fondo. Determinar si existe o no un correcto limite al poder discrecional en su ejercicio por parte de los órganos jurisdiccionales resulta bastante complejo, sin embargo, un buen indicio sobre este punto seria la discrepancia que pudiere suscitarse respecto a los resultados de la ponderación; no obstante, debemos considerar la facilidad que este hecho resulta en debates sobre aspectos morales y políticos, cuyas posturas sean muy contradictorias $\mathrm{o}$ alejadas entre sí.

En el ámbito de la ponderación judicial se pone en riesgo claramente el respeto a la legalidad, siendo el problema fundamental que plantea la ponderación, el recurrir a esta estructura argumental cuando el juez desea hacer prevalecer sus convicciones de Justicia sobre las expresadas en la ley, y en definitiva cuando se desea esquivar la ley (Fernández de Casadevante Romani, 2020). Es así que los jueces ordinarios no deberían ponderar, si no en causas muy excepcionales, y por dos razones fundamentales, en primer lugar, cuando exista un genuino conflicto de principios y donde por tanto procedería la ponderación, es decir casos en los que no existe una regulación legislativa expresa. En segundo lugar, cuando el principio o bien constitucional que hay detrás de la regla legal aplicable, entra en conflicto con otro principio constitucional que juega en sentido contrario, convirtiendo casos que tienen simple relevancia legal en casos de relevancia constitucional.

Como indicamos en la primera parte de este artículo, el tránsito que se ha tenido del Estado legal de Derecho al Estado Constitucional de Derecho, se manifiesta en la concepción de una Constitución como un programa, como una norma exclusivamente política, a una norma jurídica. Esto pone de relieve las diferencias entre la interpretación de una ley y la interpretación de una Constitución. En primer lugar, la finalidad, siendo la de la ley estar destinada a cuestiones absolutamente individuales, reglamentando la conducta individual de las personas así, la Constitución se convierte en el cauce que va a establecer las relaciones entre el Gobierno y la sociedad. En segundo lugar, respecto a su estructura normativa, la ley del supuesto de hecho tiene la subsunción y la consecuencia, la Constitución es un conjunto de directrices de principios y derechos. Por último, la diferencia en su origen, mientras la ley tiene su origen en el Poder Legislativo que viene a ser una suerte de poder constituido, la Constitución no tiene su origen en un poder constituyente y a partir de allí esa vocación de longevidad y de permanencia.

Siendo que las teorías interpretativas de la Constitución han sido desarrolladas por Jueces y Magistrados constitucionalistas, las mismas 
tienden a tener una ligera inclinación hacia el desarrollo más bien de una Constitución viviente, es decir la posibilidad de separarse de lo que dijo el constituyente histórico so pretexto de hacer una Constitución contemporánea, es el Tribunal Constitucional el principal generador de Derecho Constitucional, siendo sus sentencia aplicables y de obligatorio cumplimiento por parte de todos los ciudadanos y los poderes del Estado. Autores como Peter Häberle hablan a menudo de una sociedad abierta de los intérpretes constitucionales, señalando que la posibilidad de interpretar la Constitución está al alcance de todos; sin embargo, las interpretaciones vinculantes están a cargo del poder legislativo en primer lugar y de manera supletoria el Poder Ejecutivo, y en segundo lugar el Poder Judicial a través de los jueces en cada decisión, en cada sentencia, subyace en su decisión la interpretación constitucional. (Balaguer Callejón, op. 2004).

No obstante, es importante indicar que este ejercicio sin los recaudos necesarios como hemos puntualizado en párrafos precedentes, puede derivar en situaciones de mutación constitucional, entendiéndose no como una reforma de la constitución, sino de la inaplicación de determinados preceptos de la Constitución, ya sea mediante el desuso o sobre todo por la reinterpretación. Produciéndose esta última cuando algunos preceptos constitucionales se reinterpretan y acaban significando algo distinto a el alcance que se pretendía tuvieren, lo que derivaría en la necesidad de siempre procurar una interpretación integradora del precepto constitucional, caso contrario, el texto constitucional se interpreta de una manera distinta a aquella a la cual fue aprobada y estos son los procesos que son considerados mutaciones constitucionales, en donde estrictamente no hay una reforma pero de facto se lleva a una interpretación que conduce a que una norma constitucional no se aplique de la misa manera.

En el caso hondureño, los ejercicios de interpretación conforme, y más aún los Juicios de Ponderación, son una realidad bastante presente. Sin embargo, esto supone un choque de nuestra cultura jurídica en cuanto al sistema jurídico imperante en el país, el Civil Law o Derecho Romano, el cual sumado a que la escuela de derecho hondureña favorece la práctica de la subsunción de normas, hace bastante complicado a que los funcionarios públicos e incluso órganos jurisdiccionales distintos a la sala constitucional, realicen si quiera un ejercicio de interpretación. Situación a la que están obligados bajo Principio de Control de Convencionalidad, los órganos administrativos, por un lado, pero también los órganos jurisdiccionales y cualquier poder constituido en el ejercicio de sus atribuciones.

Esto resulta en especial importante, por cuanto en el ámbito internacional, hasta abril de 2018, Honduras había ratificado casi todos los tratados de derechos humanos pertinentes, incluidos los nueve tratados básicos de derechos humanos, quedando pendientes el Protocolo Facultativo de la Convención sobre la Eliminación de todas las Formas de Discriminación contra la Mujer, el Protocolo Facultativo del Pacto Internacional de Derechos Económicos, Sociales y Culturales y el Protocolo Facultativo de la Convención sobre los Derechos del Niño, los cuales permiten una acción coordinada entre los sectores de sociedad civil, los nacionales y los Estados, a fin de lograr un trabajo coordinado para la garantía de los mismos, y la opción de una instancia internacional supletoria. Sin perjuicio de lo anterior, resulta importante señalar que, en el ámbito nacional, nuestra Constitución es un texto claramente garantizador, 
que cuenta con una amplitud de mecanismos que persiguen investir de un verdadero carácter normativo a los derechos fundamentales $\mathrm{y}$ libertades que contiene, derechos y mecanismos de protección para hacerlos efectivos. Dichos mecanismos, además se encuentran tutelados por leyes ordinarias y secundarias, mediante la gradación de estos derechos en función de su protección individual y es que la Constitución de Honduras proclama como suyos en su Título I, Capitulo III, los principios y prácticas del derecho internacional en materia de solidaridad, autodeterminación, intervención, apreciamiento de paz y democracia, haciéndolo parte de su ordenamiento jurídico, señalando además que el Estado de Honduras proclama como ineludible la validez y obligatoria ejecución de las sentencias arbítrales y judiciales de carácter internacional.

Esta cláusula constitucional de apertura, no obstante, condiciona la jerarquía de los Tratados Internacionales en el ordenamiento jurídico interno, los cuales en elámbito hondureño tendrían una primacía por sobre las leyes hondureñas, pero en una situación de infra-constitucionalidad, al ser la propia Constitución quien determina los mecanismos para la incorporación de dichos tratados en el ordenamiento jurídico interno y en su caso, si contradice lo establecido en la Propia Constitución, se tendría que seguir el proceso que la propia constitución establece para reforma.

\subsection{El Principio Pro Persona en la Constitución Hondureña}

Sin perjuicio de lo expresado en los capítulos precedentes, la Constitución Hondureña de forma implícita contempla el Principio Pro Persona en los artículos 15, 59 y 183, en primer lugar, al dar apertura a la incorporación a las sentencias internacionales, pero además los tratados internacionales que propendan a la solidaridad humana. Sobre esto quisiéramos ahondar un poco más, ya que el propio artículo 183 de la Constitución de la Republica de Honduras, mismo que desarrolla el Recurso o Garantía de Amparo, hace una derivación a una Ley, en este caso la Ley de Justicia Constitucional, misma que en su artículo 2 establece como el proceso de interpretación en el ámbito constitucional agregando a los tratados, convenciones y otros instrumentos internacionales sobre derechos humanos vigentes en la República de Honduras a los que hace referencia el artículo 183 constitucional, las interpretaciones de los tribunales Internacionales.

Lo anterior resulta de especial importancia ya que en el ámbito americano, específicamente el Sistema Interamericano, las facultades interpretativas han sido delegadas en la Corte Interamericana de Derechos Humanos, por lo cual y recordando que Honduras es suscriptor de la Convención Interamericana de Derechos Humanos, por lo que vendría a reconocer su jurisdicción en la materia. La especial relevancia del caso reviste en que bajo una interpretación dinámica de los instrumentos internacionales, no siendo la Convención Interamericana de Derechos Humanos la excepción, los preceptos normativos se adaptan constantemente ante los requerimientos y avances constantes en el reconocimiento de derechos humanos, cosa que por ejemplo no ocurre con el ordenamiento jurídico hondureño, aun y cuando el caso Almonacid Arellano vrs Chile sentó ya las bases de control de convencionalidad desde hace ya el año 2006.

Como ejemplo de lo antes expuesto pasado el 9 de enero, la Corte Interamericana de Derechos Humanos (CorteIDH) hizo pública su opinión 
consultiva 24/17 sobre identidad de género, igualdad y no discriminación a parejas del mismo sexo, alcances que genera inmediatamente una antinomia con el artículo 112 constitucional, lo que en primera instancia supondría de acuerdo con lo establecido en los artículos 16 y 17 de la propia Constitución hondureña, la Constitución tendría que prevalecer, o bien proceder a la reforma constitucional, sin embargo, bajo la óptica del principio pro persona (pro homine) en relación con el control de convencionalidad, tendríamos que aplicar la normas que resulte más garantista.

Sin embargo, no es de desconocer que el problema antes expuesto genera intensos debates en cuanto a la aplicación de Sentencias Internacionales y los preceptos que estas recogen, sobre todo en definir si el alcance de una sentencia internacional emitida por ejemplo, por la Corte Interamericana de Derechos Humanos, a pesar que no ser específicamente en contra del Estado de Honduras, sea de obligatorio cumplimiento para el país respecto de las recomendaciones que la misma contiene en caso de identificarse que en la realidad nacional hondureña existan situaciones similares. O si bien los procesos especiales desarrollados por el Sistema Universal de Protección de Derechos Humanos, en cuanto a las observaciones generales emitidas, o recomendaciones dirigidas al Estado de Honduras, sean vinculantes y por consiguiente requieran ser consideradas al momento de la adopción de actos de autoridad por parte de los diferentes Poderes del Estado y sobre todo el Poder Judicial.

Sobre lo anterior, consideramos que cualquier disposición, interpretación u observación emitida por estos organismos especializados en el ámbito internacional aun y cuando no estén dirigidos nominalmente al Estado de Honduras, son de obligatorio cumplimiento partiendo de la premisa que existe una cesión de soberanía y competencias por parte de los Estados al momento de formar parte de estos procesos de integración en el ámbito de derechos humanos. Inclusive puede ir mas allá, por cuanto a pesar de que el país no sea parte del proceso pueda que resulte una obligación sobre todo en aquellos principios que son considerados ius cogens. Volviendo a las observaciones e interpretaciones emitidas por este tipo de órganos, no podemos perder de vista que estas versan sobre un instrumento de carácter internacional, que es aplicable directa $\mathrm{y}$ vinculantemente al país $\mathrm{y}$, por consiguiente, las observaciones y criterios emitidos en cuanto a su alcance y aplicación, generan verdaderos compromisos internacionales para todos los países que forman parte de dicho proceso.

Todo lo anterior, da como resultado un escenario optimo en donde la interpretación conforme, y especialmente los juicios de ponderación adquieren un lugar relevante en el ejercicio constitucional. Obligando a los Jueces a elegir en una suerte de esquema multinivel, sobre un variado conglomerado de preceptos contenidos en sentencias, opiniones y normas propiamente, la disposición que en atención a las particularidades del caso individualizado resulten más favorables a la garantía de la dignidad de la persona humana. Sin embargo, como hemos advertido anteriormente, este ejercicio interpretativo, obligatorio vale la pena aclarar, no se encuentra exento de la polémica y extralimitaciones. En ese sentido, analizamos por ejemplo lo indicado en el artículo 2 de la Ley de justicia Constitucional, en base al cual los órganos jurisdiccionales y más importante aún, la Sala Constitucional hondureña, proceden a emitir sentencias aditivas que trastocan el alcance, y sobre todo amplían el significado de los preceptos contenidos en la Constitución, como fue en la 
sentencia de inconstitucionalidad, emitida por la Corte Suprema de Justicia en relación con el Recurso de Inconstitucionalidad (Acumulados) No. SCO-1343=14 y 243=15, mediante el cual se autoriza la reelección presidencial en Honduras.

Otros aspectos a considerar son la pobre interpretación que se pueda hacer a partir de autores que son considerados referentes indiscutibles en el ámbito del Derecho Constitucional, y en aplicación de dichas interpretaciones se proceda a la modificación del alcance y contenido de la Constitución, dando lugar a la mutación constitucional, pero con la agravante que la misma se basaría en disposiciones doctrinarias que en el sistema de fuentes del derecho por lo general son situadas en la escala más baja y como ultima ratio, pero que sin embargo, no impide al jurista el reformar textos Constitucionales como lo fue en el caso hondureño (Viciano Pastor, 2018).

\section{CONCLUSIONES}

- La interpretación constitucional resulta de vital importancia para todos los Poderes del Estado, sobre todo en virtud que no se puede considerar a la Constitución hondureña como un proyecto social culminado que determine o contenga la solución de la mayoría de los problemas; sin embargo, el establecimiento de reglas de procedimiento claras en cuanto a los límites de la interpretación, y un rol protector e independiente por parte del Poder Constituido que se designe para la protección de la misma constitución, resultan necesarios a fin de determinar que el uso de estas herramientas necesarias en sociedades tan cambiantes. Así, el establecimiento de mecanismos de legitimación q través de una posterior consulta $\mathrm{y}$ ratificación por parte del constituyente en aquellos casos controvertidos y con poca claridad, se convierten en una alternativa bastante interesante que debe ser considerada y someterse a un mayor estudio por parte de os expertos en la materia Constitucional.

- Si bien la Constitución de Honduras no cuenta con un capítulo específico de Interpretación Constitucional, mismo que no negamos resulta necesario para el desarrollo del alcance y la forma en como los preceptos constitucionales deben interpretarse, no puede servir de justificante respecto de la mala interpretación realizada en los casos concretos antes expuestos, sobre todo en virtud que a la fecha se ha hecho un desarrollo importante y amplísimo por parte de organismos internacionales respecto a la aplicación de la interpretación sobre esquemas de proporcionalidad y ponderación que deben servir de referentes al momento de emitir sentencias aditivas.

Ante la reducción de la brecha entre el ordenamiento jurídico especifico de los países, y la normativa internacional que ha experimentado un auge trascendental en las ultimas décadas. Conceptos como control de convencionalidad, y la aplicación de principios internacionales como el pro homine, deben convertirse en ejes transversales y de obligatoria consideración por parte de los diferentes órganos administrativos cuando afecten derechos de particulares, de los diferentes órganos jurisdiccionales, pero sobre todo y especialmente por la Sala Constitucional de la Corte Suprema de Honduras, como defensor del orden constitucional en el ámbito nacional. 
- El empleo de mecanismos de interpretación y control como lo son la ponderación y la interpretación conforme, deberán ceñirse siempre a los limites establecidos por el Constituyente en el texto constitucional, debiendo tener especial precaución cuando estos deban sobrepasarse en virtud de una mayor garantía de derechos fundamentales, y garantizando la proporcionalidad en aquellos casos que un derecho deba limitarse en beneficio de otro debiendo comprobar si la medida es susceptible de conseguir el objetivo propuesto (juicio de idoneidad); si es necesaria, en el sentido de que no existan otras medidas más moderadas para la consecución de tal fin con igual eficacia (juicio de necesidad); y finalmente, si la medida es ponderada o equilibrada, es decir, si no resulta desmedida en comparación con otras por derivarse de su aplicación más beneficios o ventajas para el interés general que perjuicios sobre otros bienes o valores en conflicto (juicio de proporcionalidad en sentido estricto).

\section{REFERENCIAS}

- Atienza, M. (2013). Curso de argumentación jurídica: Representación, separación de poderes y opinión pública (1. ed.). Colección Estructuras y procesos Serie Derecho. Editorial Trotta.

- Balaguer Callejón, F. (Ed.). (op. 2004). Derecho constitucional y cultura: Estudios en homenaje a Peter Häberle. Tecnos.

- Balaguer Callejón, M. L. (1997). Interpretación de la Constitución y ordenamiento jurídico. Tecnos.
- $\quad$ Bonifacio, F. P. y López Pina, A. (DL 1987). División de poderes e interpretación: Hacia una teoría de la praxis constitucional. Tecnos.

- Canosa Usera, R. (1988). Interpretación constitucional y fórmula política. Centro de Estudios Constitucionales.

- Díaz Revorio, F. J. (2001). Las sentencias interpretativas del Tribunal Constitucional: Significado, tipología, efectos y legitimidad, análisis especial de las sentencias aditivas (1 ${ }^{\mathrm{a}}$ ed.). Derecho público: n. 10. Lex Nova.

- España. (1992). Los derechos fundamentales y libertades públicas (I): XII jornadas de estudio. Ministerio de Justicia, Centro de Publicaciones.

- Espín Templado, E. y Díaz Revorio, F. J. (2000). La justicia constitucional en el Estado democrático. Tirant lo Blanch.

- Fernández de Casadevante Romani, C. (2020). Los efectos juridicos en España de las decisiones de los órganos internacionales de control en materia de derechos humanos de naturaleza no jurisdiccional. Ciencias jurídicas y sociales. Dykinson.

- Fernández Segado, F. y Silva, J. A. d. (2003). La Constitución de 1978 y el constitucionalismo iberoamericano. Ministerio de la Presidencia, Secretaría General Técnica; Centro de Estudios Políticos y Constitucionales.

- Ferrajoli, L. y Andrés Ibáñez, P. (DL 2011). Principia iuris: Teoría del derecho 
y de la democracia : 3: La sintaxis del derecho (1a. ed. electrónica). Estructuras y procesos. Serie Derecho. Trotta. http://www. digitaliapublishing.com/a/19034

- García Manrique, R. (2007). Derechos sociales y ponderación. Fundación Coloquio Jurídico Europeo.

- Guastini, R. (2008). Teoría e ideología de la interpretación constitucional. Minima trotta. Trotta.

- Häberle, P. (2002). Constitución como cultura: (artículos seleccionados para Colombia) (1. ed.). Temas de derecho público: Vol. 66. Universidad Externado de Colombia; Instituto de Estudios Constitucionales Carlos Restrepo Piedrahita.

- Instituto Iberoamericano de Derecho Constitucional. (2009). Memoria del $X$ Congreso Iberoamericano de Derecho Constitucional: Lima, 16-19 de Septiembre de 2009 (1. ed.). IDEMSA; APDC Asociación Peruana de Derecho Constitucional.

- Jestaedt, M. y Montealegre Lynett, E. (2008). La ponderación en el derecho (1. edition). Universidad Externado de Colombia.

- Julios-Campuzano, A. de y Pérez Luño, A.E. Itinerarios constitucionales para un mundo convulso. http://repositorio.dl-e.es/viewer. vm?id=0000038659.pdf

- Kuhlen, L. (2012). La interpretación conforme a la constitución de las leyes penales. Derecho penal y criminología. Marcial Pons.
- María Luisa Balaguer Callejón. La interpretación constitucional como interpretación del derecho. Derecho Constitucional Y Cultura : Estudios En Homenaje a Peter Häberle.

- Márquez Luzardo, C. M. (2014). Interpretación evolutiva de la Constitución y teorías de la interpretación constitucional (Primera edición). Konrad Adenauer Stiftung; Universidad Católica Andrés Bello.

- Martínez Zorrilla, D. (2007). Conflictos constitucionales, ponderación $e$ indeterminación normativa. Marcial Pons, Ediciones Jurídicas y Soci.

- Mendonca, D. (2003). Los derechos en juego: Conflicto y balance de derechos. Ventana abierta. Tecnos.

- Münch, L. y Angeles, E. (1990). Métodos y técnicas de investigación para administración e ingeniería (2 ed.). Trillas.

- Niembro Ortega, R., Pomed Sánchez, L. y Trejo Osornio, L. A. (2012). Jurisprudencia del Tribunal Constiticional Español. Biblioteca Porrúa de Derecho Procesal Constitucional: Vol. 60. Porrúa; Instituto Mexicano de Derecho Procesal Constitucional.

- Parejo Alfonso, L. (DL 1990). Constitución y valores del ordenamiento. Centro de Estudios Ramón Areces.

- Queralt Jiménez, A. (2008). La interpretación de los derechos: Del Tribunal de Estrasburgo al 
Tribunal Constitucional. Centro de Estudios Políticos y Constitucionales.

- Saiz Arnaiz, A. y Fabián Caparrós, E. A. (2000-<2004>). Responsa iurisperitorum digesta ( $1^{a}$ ed.). Ediciones Universidad de Salamanca.

- Viciano Pastor, R. (2018). Cuando los jueces declaran inconstitucional la Constitución: la reelección presidencial en América Latina a la luz de las últimas decisiones de las Cortes constitucionales. Anuario Iberoamericano De Justicia Constitucional(22), 165-198. https://doi.org/10.18042/cepc/aijc.22.06

- Vigo, R. L. (2017). La interpretación (argumentación) jurídica en el estado de derecho constitucional (Primera edición). Tirant lo Blanch. Jueces y argumentación jurídica. Consejo de la Judicatura Federal, Instituto de la Judicatura Federal, Escuela Judicial; Tirant lo Blanch.

- Villaverde Menéndez, I. (1997). La inconstitucionalidad por omisión. Monografía. McGraw-Hill, Interamericana de España.

- Wolfe, C., Rubio de Casas, M. G. y Valcárcel, S. (1991). La transformación de interpretación constitucional ([ $1^{\text {a }}$ ed.]). Civitas. 
